\title{
German Lexical Borrowings in the Chronicle of Barkułabava and in the Memoirs of Theodore Jeŭłašeŭski
}

\author{
BY
}

\author{
R. J. PATRY-TAMUSHANSKI
}

\section{Introduction}

The manner in which German words appeared in Middle Byelorussian (15th-17th centuries) is a complicated question.

The academician Je. F. Karskij, the first to study the more important foreign lexical components in the Byelorussian language, held that many of the words of German origin came into Byelorussian through the medium of the Polish language. In addition to this, Karskij also admitted the possibility of some words being borrowed directly from the German, or by the medium of Yiddish. He believed this to be so in the case of many words connected with commerce, the smith and carpentry trades, for these activities were usually in the hands of the Jewish population. ${ }^{1}$

The Teutonic Order began its colonising in the Baltic lands from the 13th century. Their influence is felt in the language from the second half of the 14th century. Direct borrowing from German would most likely concern these earlier times, when there existed direct trade relations between the population of Byelorussia's northern cities and the German merchants of Riga and Gotland. Połack and Viciebsk in due course also became Hanseatic cities. ${ }^{2}$

In the Baltic lands at one time a Low German dialect was to a certain extent the colloquial and trading language, and even the grand dukes of Lithuania made use of this dialect, in addition to the local Byelorussian language, in their various dealings with Livonia. Trade agreements and documents of that time were often drawn up in the form of the corresponding German documents. ${ }^{3}$

The earlier contacts between Byelorussians and Germans were by the spoken word, later by the written and printed word. As a result of Magdeburg law a number of Germans came to Byelorussia. This furthered more direct contacts and more direct borrowing of German

1. Je. F. Karskij, Belorusy. Vvedenije $k$ izučeniju jazyka i narodnoj slovesnosti, vol. I, Vilna, 1904, p. 159.

2. I. I. Cartko, 'Praniknieńnie hiermanizmaŭ u leksiku starablełaruskaf movy', Viesci Akademii Navuk BSSR. Seryja hramadskich navuk, 1, Minsk, 1966, p. 121.

3. A. Ja. Bachańkoŭ et al. (eds), Histaryčnaja leksikatohija bietaruskaj movy, Minsk, 1970 , p. 117. 
vocabulary. The colonising Germans who came were specialists in the field of military matters, tradesmen, merchants and agriculturists. ${ }^{4}$

None the less, we should not underestimate the role played by Polish in bringing German words into the Byelorussian language of the 16 th and 17 th centuries. Karskij and later authors who studied this problem agree that practically all the Germanisms found in Middle Byelorussian were also found in the Polish language of the same time and that these German loan-words in both languages are either identical or similar in phonetic and morphological structure. ${ }^{5}$

The influx of Germanisms seems to coincide with the appearance of Polonisms in Byelorussian. However, we can still consider them a separate lexical category, because often semantically, as well as phonetically, they can be clearly recognised as words derived from German.

Jews began to come to Byelorussian territory in the 13 th century. They settled in many Byelorussian towns and practised commerce, trades and medicine. That they became closely involved with the Byelorussian population can be shown by the fact that their Yiddish language, in effect a dialect of medieval German with an admixture of Hebrew and other elements, took on a special Byelorussian 'colour' - often showing akańnie, ciekańnie and dziekańnie, as well as in vocabulary. ${ }^{6}$ Phonetic criteria have been employed to establish the medium of Yiddish, but, since many words have very similar or identical forms in Polish, the evidence can hardly be considered conclusive.

The Byelorussian language adapted the German words to its own phonetic system. The fact that a good number of these German loanwords still remain in modern Byelorussian is evidence that they are now completely integrated into the language.

Two very interesting works of late 16 th and 17 th century Byelorussian are quite similar in character and embrace roughly the same period of time. They are the Chronicle of Barkutabava and the Memoirs of Jeŭłašeŭski, Assessor of Navahrudak. The two works, however, differ both in style and content. In many ways they complement one another. The Chronicle gives us much information about village life and ordinary people, who are almost entirely ignored in the Memoirs.

The Chronicle of Barkułabava was written by an unknown author - an inhabitant of the village of Barkułabava, in the region of Bychoŭ, in the present-day Mahiloŭ province in the eastern part of Byelorussia. He probably belonged to the lower Orthodox clergy and was closely associated with the life of the ordinary villager and peasant. The Chronicle describes events taking place in the area of Mahiloŭ, Połack and, of course, in the environs of the village of Barkułabava itself, between the years 1563 and 1608 , and after a break gives a short incomplete entry for 1633 .

4. tbid.; Cartko, op. cit., p. 126. 5. A. I. Zuraŭski, Historyja bietaruskaj litaraturnaj movy, I, Minsk, 1967, pp. 118-19;

6. U. Weinreich, 'Yiddish and Colonial German in Eastern Europe: the differentlal Impact of Slavic', American Contributions to the Fourth International Congress of Slavicists, The Hague, 1958, pp. 372-4. 
The work dwells in detail on facts and episodes of local life, nature, the conditions of peasant life, and interesting happenings which took place in Barkułabava. Ordinary people and village life interest the unknown author more than the affairs of the nobility, political events and religious polemics. Abaut these events he often writes inaccurately regarding names and dates. ${ }^{\top}$

In often picturesque words, the author reveals the moods and feelings of his contemporaries. The language of the Chronicle is the living Byelorussian as spoken by the ordinary people, and is remarkably free from Polish and other foreign influence. It may well be said that the linguistic tradition of Skaryna is further developed in the Chronicle. Comparatively few lexical Polonisms are found, the author often using Byelorussian words to enrich the concepts which are rendered by Polonisms in other documents. ${ }^{8}$ None the less, we shall see that it still contains a number of German loan-words.

The Memoirs of Theodore Jeŭłašeŭski, Assessor of Navahrudak, cover the period 1546-1604. Jeǔlašeŭski was of minor noble stock, who were 'interested in Ruthenian writing, made translations, propagated them, wrote their own works, occupied various elected civil positions and at that time were ready to fulfil the desires of the lords, the patronage of whom was often more important for the lesser nobility than state service'. ${ }^{9}$

We have little information about Jeŭłašeŭski other than what he tells us himself. He was born on 7th February 1546 in Lachavičy, in western Byelorussia, into an Orthodox family. Later he became a Calvinist but remained on good terms with both Orthodox and Catholics. He gained much by self-education, and because of his deep knowledge of law he enjoyed the admiration of his friends. He made a name for himself as a jurist and in 1579 was sent to parliament. Jeŭłašeŭski took part in the drafting of the Rules of High Court (Tribunal). He died in $1604 .^{10}$

Shortly before his death he began to write his memoirs, which his successors called the Dzionnik. This work, which is based on concrete experiences, provides us with a whole gallery of his contemporaries, a vivid picture of the social life of the Byelorussian nobility in the second half of the 16th century, and some aspects of political events in Byelorussia during an important and interesting epoch of her history.

The language of the Memoirs is Middle Byelorussian, as used in state documents and contemporary works of literature. However, no

7. V. V. Barysienka, Historyja bietaruskaj dakastryčnickaj litaratury, I, Minsk, 1968, pp. 261-2.

8. M. I. Karniejeva-Pietrułan, 'Mova Barkułabaŭskaha letapisu', Pracy Instytuta movaznaŭstva AN BSSR, III, Minsk, 1957, pp. 94-111.

In the composition of the Chronicle, in addition to the author's own experiences, other elements are incorporated which were copied from other sources. Although in the copied sections one can recognise a more polonised and 'bookish' language, these sections do not contain a greater amount of Germanisms than the original writings cf the author himself.

For further information on the Chronicle and its language see V. Volski, Narysy pa historyi bietaruskaj litaratury epochi feadalizma, Minsk, 1958, pp. 70-5; Barysienka, op. cit., pp. 260-8; Zuraŭski, op. cit., pp. 342-6.

9. Karskij, op, cit., III, 1921, D. 108.

10. Barysienka, op. cit., pp. 254-5. 
other example of Byelorussian writing of the time illustrates in such a degree the influence of the Polish language as do the Memoirs. Jeŭłašeŭski's style is smooth but there is a definite leaning towards complicated sentence structure, very much revealing his membership of the legal profession. ${ }^{11}$

In examining the German lexical borrowings in these two works one finds that the Memoirs have more German loan-words than the Chronicle, that there is a certain number of words found in both works, as well as some peculiar to each.

The texts used in this study are:

'Barkulabovskaja letopis' [ed. A. N. Malcev], Archeografičeskij ježegodnik za 1960 god, Moscow, 1962, pp. 291-320.

'The Memoirs of Theodore Jeŭłašeŭski, Assessor of Navahrudak (1541-1604)' [trans. and annotated by A. Nadson], JBS, I, 4, London, 1968, pp. 269-348.

Abbreviations used:

C - Chronicle of Barkułabava

$\mathrm{J} \quad$ - Memoirs of Theodore Jeŭłašeŭski

ahd. - Old High German 1. - Latin

and. - Old Low German mb. - Middle Byelorussian

cs. - Common Slavonic mhd. - Middle High German

cz. - Czech

d. - Dutch

fr. - French

G. - (modern) German pol. - Polish

t. Transliteration:

mnd. - Middle Low German

Mod. B. - modern Byelorussian

nd. - Low German

The standard academic transliteration of Cyrillic texts is employed. The Middle Byelorussian phoneme $\mathrm{kr}$ is rendered by $g$.

II

Vocabulary

ARCYBISKUP, BISKUP '(Catholic) archbishop, bishop'

B-295 mnoho bylo biskupov, panov rad

B-305 arcybiskup l'vovskij, biskup luckij

J-290 na mestcu korolevskom arcybiskup Jakub Uchanskij sidel

$\mathrm{J}-298$ v majetnosti biskupstva vilenskoho

$\mathrm{J}-298$ a biskup on cnotlivyj

pol. cz. biskup from ahd. biscof.

BALKA 'beam, rafter'

J-294 Januš mlynar stal na krajnej balce

pol. balka from nd. balke; G. Balken.

BALVER (in other documents also BARVER, BARBER) 'physician'

$\mathrm{J}-318$ kazal židovi balverovi mazati sobe

pol. balwierz from mhd. and G. barbier, 1. barba. Note the partial dissimilation of $r$.

11. For full information about the Memoirs as well as a parallel translation of the text see A. Nadson, 'The Memoirs of Theodore Jeưłašeŭski, Assessor of Navahrudak (15411604), JBS, I, 4, London, 1968, pp. 269-348. 
BLOK 'block, lifting mechanism'

$\mathrm{J}-290 \mathrm{u}$ voze velikom, no to zahotovanom $\mathrm{z}$ blokami

pol. blok from mhd. and G. block

BUDOVATI 'to build'

B-317 zbudoval car Dmitr... kostel

$\mathrm{J}-326$ zbudoval izbe sobe sam

pol. budować from mhd. buode

BUNT 'revolt, rebellion'

B-317 velikij bunt zabite, krovoprolite

J-306 u spikneniu i zbuntovaniu l'udskom zhinul

pol. bunt, mhd. bunt. There is no phonetic change in this word, but a notable semantic shift. Mhd. bunt is 'band, tie, league, alliance, confederacy'.

COFNUTI 'to retreat, go back, draw back, withdraw'

J-322 nazad se cofnuvšy, po doroze i chrustok zostavili

pol. cofać, cz. coufati. Miklosich derives this from mhd. zofen, Brückner from the Bavarian zaufen, zurückzaufen.

CYBUL'A 'onion'

B-314 botvinje, cybul'a, maki

pol. cybula, mhd. zwibolle, zibolle, 1. caepulla

DACH 'roof, shelter'

J-302 kotoroho dach pripuščonyj byl

pol. cz. mhd. dach

D'AKOVATI 'to thank'

J-330 D'akuju ja Tobe, Bože moj

pol. dziękować, cz. děkovati; mhd. danken, and. danc

DRABINA 'step ladder; (type of) wagon'

$\mathrm{J}-286$ až drabinu $\mathrm{z}$ vesi prinesši

pol. cz. drabina, mhd. trappe, trappen, treppe

FAL'S 'untrue, false, dishonourable, in error'

B-301 listy fal'šivyje pisati

J-298 movami svoimi fal'šivymi nas ščipali

pol. fatsz, cz. faleš, from mhd. valsch, vals, l. falsus

FOLGOVATI 'to follow, succeed, ensue'

$\mathrm{J}-288 \mathrm{mi} \mathrm{v}$ doroze $u$ folgovat' račil

pol. folgować, old cz. folkovati, from mhd. volgân

FOLVARK 'estate, small farm, property situated before the city'.

J-310 folvark l'achoveckij Pavl'ukovskij

pol. folwark, old pol. forwark, old cz. forberk, folvark from mhd. vorwërc.

Note here another case of $r-l$ dissimilation.

FORTKA 'gate, door'

B-305 prijechali do kostela ... do fortki

J-304 i perepustivši Volodimira za fortku

pol. forta, mhd. phorte, phorze, l. porta

FRASOVATI 'to disturb, make sad, worry' 
FRASUNOK 'worry, care'

J-288 velce se $z$ frasovala i zdorove sobe popsovala

J-288 po pervich chorobach moich i frasunkach svoich značne zdorovia urvala

pol. frasunek, cz. fresovati, mnd. fressen, vra3. There is a semantic shift here. Fressen means 'to eat' (of animals). There is little to suggest 'worry' in the German usage of this word.

FRIMARK 'exchange'

J-290 frimarkom za Svisloč pustil

pol. frymark, cz. frimark, mnd. vrîmarket

GRABIA 'count, baron'

$\mathrm{J}-330$ pan grabia z Tenčyna

According to Miklosich, the oldest Slav borrowings are from the ahd. grāvjo, pol. grabia, cz. hrábě, mnd· grâve

GRUNT 'ground, foundation, justification, reason'

B-269 za Pečerskoje na tetoj storone grunty

B-308 na vlasnom grunte

J-316 zašli byli grunt Vereščačin

pol. cz. mhd. grunt

GVALT 'force, punishment, obligation, violence'

B-299 velikije doždy gvaltovnyje domy podryvali

B-305 prave gvaltom, slyše, primušano do prisiahi

$\mathrm{J}-284$ povetre gvaltovne $\mathrm{v}$ meste

$\mathrm{J}-288$ až juž gvaltovne mia v domu zlomalo

B-314 zmordovanuju, gvalčonuju, ledvej živuju

Note the adjectival derivatives. Gvalčonaja bears the identical connotation as the $\mathrm{G}$. vergewaltigt (B-314).

pol. gwalt, cz. kvalt, mhd. gewalt. Karskij derives this from the Yiddish giewatt.

HAMOVATI 'to stop, hinder, restrain'

J-304 prijechavši, hamaval jeho.

pol. hamować, mhd. hemmen

HETMAN 'commander-in-chief'

B-297 pan Andrej Hudovskij hetmanom byl

pol. hetman, old cz. hýtman, hajtman, hejtman, hutman, nd. hovetman, mhd. heuptman.

Miklosich derives this from G. Hauptmann.

HURMOM 'en masse, noisily'

B-303 rečy začatyj končiti i razjechalise, a hurmom z narekanjem J-328 zeštja tož z sveta l'udej zacnych hurmom

pol. hurmem, mhd. hurm 'enemy attack'. This word is used adverbially in Middle Byelorussian. There is here also a semantic shift.

JALMUŽNA 'alms'

$\mathrm{J}-312 \mathrm{v}$ mesto jalmužnej mel otrymati prezysk na dobrach panskich pol. jalmużna, cz. almužna, mnd. almuosen, ahd. alamousan, 1. eleemosyna, Greek eleemosyne 
JARMARK, JARMAROK 'bazaar, fair'

B-299 kupcom torhi albo jarmarki pootmeniali

B-308 jako jarmaročnoho času kreščena byla u vannie pohružanna J-320 šel do Lucka, v jarmarok prave

Note the interesting adjectival derivative in B-308. In J-320 we have an example of a svarabhakti vowel. We also have a semantic change here. Whereas mhd. jârmarket means 'annual fair', in Byelorussian it takes on the additional gloss of any 'bazaar'. pol. cz. jarmark

KANCLER 'chancellor'

B-302 z jeho milostju panom kanclerom

J-300 pan Zamojskij, kancler

pol. kanclerz, G. Kanzler, 1. cancellarius

KAPUSTA 'cabbage'

B-314 kapusta, botvinje, cybul'a, maki

ahd. chapuz, chabuz, pol. cz. kapusta, 1. composita

KASTAL'AN 'steward'

B-305 prislali pana Precbica, kaštal'ana kameneckoho

J-288 pan Mikolaj Pal'vis, kaštal'an menskij

pol. kasztelan, mhd. kastelân, Italian castellano, 1. castellanus

KELICH 'cup, chalice, goblet'

B-305 v kelichu vino $u$ krov barzo špetnuju smrodlijuvu obernulasia pol. kielich, old. cz. kalich, kelich, mhd. kelch, kelich, l. calix

KL'ASTOR 'monastery'

$\mathrm{J}-304$ hde velkost' l'udej pešich v $k l^{\prime}$ aštore byla

pol. klasztor, cz. klášter, klášteř, mhd. klôster, 1. claustrum

KOST 'cost, expenses'

B-316 koštom velikim vybranych

B-318 ubiory koštovnyje odeli

J-316 ves' koštovna ot Hniezna

pol. koszt, mhd. koste - there is here a narrowing of meaning.

Besides meaning 'worth, expenses', koste also means 'food, victuals, provisions'.

KRAMA 'shop'

B-306 mesto pobožnoje, domy, kramy

pol. kram, mhd. krâm

KUCHMISTER 'chief cook'

J-296 kuchmistr Kračok

pol. kuchmistrz, cz. kuchár̆. This word is in effect a compound word coming from mhd. kuchen, küche and mhd. meister.

\section{LANCUH 'chain'}

B-319 v lancuh kazali posaditi

J-316 mel pri sobe ze dvadciat osob lancužno

Note lancužno in J-316 'twenty persons in his retinue', a figurative use of the word. Miklosich derives this word from ahd. lanici, mhd. lan, lanne 'chain'. A similar word lantzuc exists in mhd. This is a war march of the whole land. In other Middle Byelor'ussian documents lancuh is to be found with this meaning. 
LOTR 'bandit'

B-305 tych lotrov, mitropolita, ich vladyk ne vidali

pol. łotr, cz. lotr. Miklosich derives this from ahd. lotar, Matzenauer from mhd. loter. Here again we have an example of a semantic shift. In ahd and mhd. the word means 'good-for-nothing, Taugenichts'. In Czech and Polish the meaning has already changed to 'rascal, bandit, thief'.

MARGRABIA 'margrave'

J-300 margrabia brandenburskij

mhd. marcgrâve; pol. margrabia, cz. markrabě

MARSALOK 'marshal'

B-302 na samym roku maršalka obrali duchovnyje

J-294 maršalku mozyrskomu

J-300 maršalkoval meži poslami naš litvin

Note the svarabhakti vowel, as well as the verbal derivative.

pol. marszałek, cz. maršál(ek), mhd. marschalc

MIL'A 'mile'

B-295 na kolkonadcat' mil' stojati

J-294 jechati mili z poltretej

pol. mila, cz. míle, mhd. mîle, 1. milia

MLYN 'mill'

J-294 u mlyna l'achoveskoho

J-294 hde Janus mlynar stal

$\mathrm{J}-322 \mathrm{u}$ toho mlynka

J-294 gives us an example of a nominal derivative mlynar 'miller'. In J-322 we have an example of a diminutive mlynok, with a svarabhakti vowel.

pol. młyn, cz. mlýn, ahd. mulîn, fr. moulin. Machek, s.v. derives this from the old German back to med. 1. molina.

MORD 'murder, homicide'

B-314 zmordovanuju, gvalčonuju, ledvej živuju

B-317 ich pobili, pomordovali

B-317 vilikoje zabijstvo, morderstvo, krovoprolitstvo

B-319 bivši i mardovavši

All contexts here show the word in a derived form. B-319 gives a striking example of Byelorussian akañnie, which seldom manifests itself in Middle Byelorussian documents due to strict adherence to an orthography based on Church Slavonic. pol. mhd. mord.

MUR 'sione wall, partition, protective separation'

B-302 v cerkvi sobornoj murovanoj

B-307 tam že jeho zamurovavši

$\mathrm{J}-304$ pod mury skladajuči se

J-314 položene zamku, mesta, vod, mostov, murovanych

pol. mur, mhd. mûre, miure, ahd. mûra, mûri, nd. mur, 1. murus

MUSITI 'to be obliged to, must, have to'

B-310 končiti byste museli

B-305 prymušano do prysiahi 
J-288 a ja ješče oseni tot musilem Varšavu navediti

B-305 shows a derived form. pol. musieć, cz. musiti, mhd. müeзen

OFERA 'offer, sacrifice'

B-304 ot toho namestnika patriarchovoho oferovan

pol. ofiara, cz. ofëra, ahd. opfar, l. offere

OLSTRO 'holster, pistol case'

$\mathrm{J}-286$ zhinula mi byla ručnica korotka $\mathrm{z}$ olstrem

pol. olstro, nd. holster

PALAC 'palace'

B-316 hrozno vdarili na palacu samoho car'a

cz. palác, mhd. palas, palast, fr. palais, l. palatium

PLAC 'square'

B-309 ale oni plac otrymali

pol. plac, mhd. platz

PL'AGA 'misfortune'

J-282 značnymi pl'agami otvražajuči

pol. plaga, mhd. plage, l. plaga

PLENDROVATI (in other documents PLUNDROVATI) 'to lay waste, to plunder'

B-318 zamki malyje vyplendrovali

$\mathrm{J}-320$ splendroval majetnosti pana Kalinovskoho

pol. pladrować, plondrować, mhd. plundern

PULHAK '(type of) musket'

B-306 pulhakov velmi pri sobe množestvo mel

J-304 tedy mohut jeho $z$ pulhaku zabiti

This word presents an example of a Slav-German compound.

pol. pót- 'half' and G. Hacken... pol. puthak, G. Halbhaken 'hand fire-arm'

PUSKA '(type of) weapon; box'

B-319 puška sažni tri i bolej

pol. puszka, cz. puška, ahd. buhsa, Greek pyxis

RADA 'counsel, advice, administration'

B-295 panov rad

J-288 pišu i detkom svoim radu moju

mhd. rât. Also derived from rât: UR'ADNIK 'official'

B-297 ur'adnik barkulabovskij

RATOVATI 'to rescue, save'

RATUNOK 'rescue'

B-310 a inšoho ratunku na tot čas otčizne našoj ne vidimo

$\mathrm{J}-292$ me penezmi byl poratoval

$\mathrm{J}-292$ me značne ratoval

$\mathrm{J}-296$ o takovych ratunku myslila

pol. ratunek, ratovać, cz. retovati, mhd. retten, rettunge

RATUS 'town hall'

B-301 ne tolko na ratušach i pri rynku

pol. ratusz, cz. rathús, a compound word, mhd. râthûs 
RICER 'knight, cavalier'

B-295 i vsi rycerstva vsich zemlej

$\mathrm{B}-296 \mathrm{u}$ sprave rycerskoj vojennoj

B-297 rycer i vojennik dobryj

$\mathrm{J}-294$ dobrotlivyj i rycerskij pan

$\mathrm{J}-294$ panove i rycerstvo

pol. rycerz, cz. rytír, mhd. rîter

ROTA 'squadron'

B-318 otsel'a z Litvy, Ruckij z rotoju

pol. rota, mhd. rotte, from old fr. rote

ROTMISTR 'squad leader or commander'

B-297 rotmistr krolevskij, vojt disenskij

J-302 z'jechali se rotmistrove i žolnere

Compounded from rota and mistr. pol. rotmistrz, old cz. rotmistr, mhd. rotemeister

RYCHLYJ 'prompt, swift'

B-310 rychlym pribytem

$\mathrm{J}-294$ ne rychlo $\mathrm{z}$ niej ukazal se

pol. rychty ... Brückner derives this from G. röcheln, rütteln which cannot 'rattle, vibrate'. An example of transferred sense!

RYNOK 'market'

B-301 ne tolko na ratušach i pry rynku

J-314 v mestečku v Kozim rynku

pol. rynek, cz. rynek, rynk, mhd. rinc, ring. Note the intrusive vowel, as well as the semantic shift. Rinc in medieval German meant 'ring, circle, place, group of persons, assembly, of lawyers, etc'.

SKARB 'treasure'

B-302 panom podskarbom

B-306 a kramov $\mathrm{z}$ velikimi skarbami 400

$\mathrm{J}-284$ pisar'a v skarbe korol'a

pol. skarb, ahd. scërf (d. scherf). Brückner curiously derives this word from G. Korb.

STODOLA 'granary, warehouse'

$\mathrm{J}-286 \mathrm{z}$ voza mojeho $\mathrm{v}$ stodole

pol. stodoza, cz. stodola, ahd. stadal

SACOVATI 'to evaluate, tax'

J-302 šacujučy tu majetnost'

pol. szacować, mhd. schätzen

SIKOVATI 'to put into order, equip, put into formation'

$\mathrm{J}-322$ sikovalise $\mathrm{v}$ pol'u

pol. szykować, mhd. schicken

SKODA 'damage, shame, disadvantage'

B-301 a krivdy škody ne činily

B-302 Nikoli ni v čom škoditi ne majet

J-282 dla uterpenia velikich prihod, trudnostej, škod

J-298 čarami preškoditi to usilovali

$\mathrm{J}-300$ neškodene byl jeho milost' 
Derivations: škoditi, preškoditi, neškodenyj.

pol. szkodz, cz. škoda from ahd. scado. Germanic cognates: Scandinavian skadha, English scathe

SL'ACHTA 'nobility'

B-295 vsia šl'achta choruhavnaja

B-310 volnost'am šl'achetskim

J-286 nevesta stara šl'acht'anka

J-294 melem spravu tam z šl'achetnoho muža

pol. szlachta, cz. šlachta, from ahd. slahte 'slaughter, killing, race, origin, tribe, kind, type'. In compounds in G. the word can take the meaning of 'aristocracy', especially in later forms, v.g. Geschlechtsname, Geschlechtsbaum, Geschlechtsfolge.

SPEG 'spy, scout, informer'

B-304 zovučy jeho špegom z Belhoroda

J-306 prez falšivyje špegi z nevelikim l'udom pripal

pol. szpieg, old cz. špehér, špehýr̆, from mhd. spëhaere

SPETNYJ 'ugly, disgusting'

B-305 v kelichu vino u krov barzo špetnuju smrodlivuju overnulasia pol. szpetny, cz. špatný from mhd. spat 'dry, arid, parched'

In Middle Byelorussian the meaning has been extended.

STRAFOVATI 'to tax, demand'

J-302 štrafujuči o to zacnoho pana

pol. strofować, from mhd. strâfe

STUKA 'piece, trick'

J-288 divne štuki vyrežal

pol. sztuka, from mhd. stücke, stuke. Here the sense of the word is widened in Byelorussian to mean 'slyness, trick' as well.

STURM 'storm, disturbance, attack'

B-297 mocno i ochotne šturme meli

pol. szturm from mhd. sturm

SUKATI 'to seek, to look for' (here OSUKATI 'to deceive')

J-318 panov v ich spravach ošukovali

ošukovati is derived from šukati. pol. szukać, cz. šukati, from nd. soeken, G. suchen

SVAGER 'brother-in-law'

J-324 z pomoč'u Korsaka, švagra svojeho

pol. szwagier, cz. švagr from mhd. swâger

SVANKOVATI 'to falter, be deficient, lack'

J-322 u nebačnych l'udej na slave švankovati musel

pol. szwankować, G. schwanken

TAL'AR 'thaler' (unit of currency)

B-308 žy to kupovali čvert po tal'aru

pol. taler, talar from G. Taler

TANEC 'dance'

$\mathrm{J}-300 \mathrm{v}$ nocy $\mathrm{z}$ tancami čineno

Note the intrusive vowel (svarabhakti). pol. taniec, mhd. tanz 
TRAFITI 'to happen, come upon, hit'

J-282 udavši se na službu, trafiali me se taki pany

pol. trafić from mhd. treffen

TRUNA 'coffin, box'

J-292 ješče bez truny na stole pri stene

pol. trumna, mhd. truhe

URUHOVATI 'to mock, insult'

B-317 popov moskovskich uruhovali

pol. uragać. Brückner and Bułyka derive this from G. rügen 'to reprove, blame, (archaic) punish'.

VAHA 'weight, importance'

J-304 hamoval jeho, rozvažajučy tot neščastnym pripadok

Rozvažati 'to consider, ponder, weigh in one's thoughts' is a derivative of vaha, pol. waga, ahd. waga, mhd. wâge

VANNA 'bath tub'

B-308 jako jarmaročnoho času kreščena byla, u vanne pohruženna pol. wanna, G. Wanne

VAROVATI 'to make a condition, to beware, to confirm'

VARUNOK 'condition'

B-301 u vsemu jepiskopstvu cerkvi vostočnyja rimskija varujem i upevniajem

B-304 v druhoj izbe varovavši sobe tož

B-310 do varunku i bespečenstva

$\mathrm{J}-284$ abym se perel'aknenia varoval

pol. warować, mhd. wâren 'to watch over, preserve'

VOJT 'major'

B-297 rotmistr krolevskij, vojt disenskij

$\mathrm{J}$-304 Avguštyna, vojta vilenskoho

J-304 i dachu vojtovskoho

Note adjectival derivative in $\mathrm{J}-304$.

pol. vójt, cz. vojt, mhd. voget, vogt, voit, from l. vocatus

ZEGAR 'clock'

B-302 hodin zegarovych bolš p'ati pod poluden'

J-306 na calom zegaru hodiny 14

J-326 šest' hodin zegarovych

Note adjectival derivative in B-302 and J-326.

pol. zegar, from mhd. zeiger 'pointer, signal, hand of a clock, clock'. Miklosich derives this from G. Seiger, from sigen 'sink', originally a sand or water clock.

ŽEGNATI 'to bid farewell, make the sign of the cross'

B-305 prijechal do kniažati, požegnal

$\mathrm{J}-296$ požegnali poslove korol'a

pol. żegnać, cz. žegnati, from mhd. segenen, cf. Machek, s.v. l. signare

ŽOLNER 'soldier'

B-314 na tot čas žolneri ležali

B-317 nemilostivoje žolnerstvo 
$\mathrm{J}-302 \mathrm{z}^{\prime}$ jechali se rotmistrove i žolnere

Note nominal derivation in B-317. pol. żołnierz, cz. žoldnér̆.

From mhd. soldenaere 'mercenary soldier'.

\section{III}

Linguistic observations

\section{A. Semantics:}

1. German loan-words from the Memoirs and the Chronicle still in use in Modern Byelorussian:

achviara, balka, biskup, budavać, bunt, cybula, dach, dziakavać, drabina, falš, falvarak, fortka, hrunt, kancler, kapusta, kaštalan, košt, krama, łancuh, łotr, maršał, mila, młyn, mur, musić, plac, puška, rada, ratavać, ratunak, rycar, rynak, skarb, škoda, šlachta, štraf, šturm, šukać, švahier, taniec, trapić, truna, vaha, vanna, vojt, žahnacca, žaŭnier.

2. It is possible to place the German loan-words from the Memoirs and the Chronicle into these semantic categories:

a) People and occupations: arcybiskup, biskup, balver, grabia, hetman, kancler, kaštal'an, kuchmistr, lotr, maršalok, mlynar, ricer, rotmistr, šl'achta, špeg, švager, uradnik, vojt, žolner.

b) Military terminology: hetman, maršalok, olstro, plundrovati, pulhak, puška, rota, rotmistr, šikovati, špeg, šturm, žolner.

c) Trade and commerce: frimark, jarmarok, košt, krama, rynok, šacovati, tal'ar.

d) Buildings, building trade, household: balka, blok, budovati, dach, drabina, folvark, fortka, grunt, $\mathrm{kl}^{\prime}$ aštor, lancuh, mlyn, mur, palac, plac, ratuš, stodola, truna, vanna, zegar.

e) Farming and agriculture: cybul'a, folvark, grunt, kapusta, stodola.

f) Christianity: arcybiskup, biskup, jalmužna, kelich, kl'aštor, ofera, žegnati.

g) Miscellanea et abstracta, verba: bunt, cofnuti, d'akovati, fal'š, folgovati, frasunok, gvalt, hamovati, hurmom, mil'a, mordovati, musiti, pl'aga, ratunok, rozvažati, rychlyj, škoda, špetnyj, štrafovati, švankovati, tanec, štuka, uruhovati, varovati. 
When we examine and compare corresponding words in two different languages, we may see that some words have identical meanings in both languages. Loan-words differing in meaning may have an expanded meaning in the borrowing language, or the sense may have been narrowed. There are also words which have transferred sense or use in the borrowing language.

Words in the Chronicle and Memoirs identical in meaning in both German and Middle Byelorussian:

balka, balver, biskup, blok, buda, cybul'a, dach, d'akovati, fal'š, fortka, frimark, grabia, grunt, gvalt, jalmužna, kancler, kelich, kl'aštor, košt, krama, kuchmistr, margrabia, maršalok, mil'a, mlyn, mur, musiti, ofera, olstro, palac, plundrovati, pulhak, puška, ratunok, skarb, šacovati, škoda, špeg, švager, tal'ar, tanec, truna, vanna, vojt, žegnati, žolner.

The above list accounts for more than half the words. Of the words not agreeing in both languages as found in the examined texts, the following are worthy of note.

Words having an expanded meaning in Middle Byelorussian:

mur in mb. is 'stone wall, partition, protective separation'; mûr in mhd. 'wall'.

frasunok 'sadness, turbation', in addition to its infrequent use in mb. as 'food'; mhd. vra3 'food (of animals)'.

štuka besides meaning 'piece' or 'bit', also means 'joke' or 'trick', a meaning not held by the mhd. stücke.

jarmark means any 'bazaar' or 'fair' not just the 'annual fair' implied by the mhd. jârmarket from which it is derived.

Words having a transferred sense or use:

bunt $<$ mhd. bunt 'band, tie, lelague, union, alliance'. Perhaps we have an example here of lexical contagion: an alliance that is out to do no good.

hurmom 'en masse, noisily'; in mhd. hurm is 'enemy attack'.

lotr 'bandit'; in mhd. loter, from which the Byelorussian word is derived, means 'good-for-nothing'.

rynok 'market place, market'; cf. mhd. rinc, ring 'ring, circle, arena' etc.

šl'achta 'nobility', s.v.

špetnyj 'ugly, disgusting' is derived from mhd. spat 'dry'. 
B. Phonetics:

The German loan-words found in the Memoirs of Theodore Jeŭłašeusski and the Chronicle of Barkułabava offer us some material for observing how the Byelorussian language modified the German words to suit its own phonetic system. One must here bear in mind that these words do not comprise all the German loan-words in Middle Byelorussian, and thus it is not possible to indicate all phonetic adaptations in this small study.

Vowels: The vowels $a, e, i, o, u$ are common to both languages.

$a$ common to both German and Middle Byelorussian: dach, jalmužna, šl'achta, fal'š, jarmark, krama

$a>0$ : stodola, škoda

$a>$ e: špetnyj

$e$ common to both German and Byelorussian: špeg, žegnati

e $>a$ : ratunok

$i$ common to both languages: biskup, ricer

o common to German and Middle Byelorussian: folgovati, folvark, ofera, žolner, mordovati, lotr

$o>$ : biskup

$o>a: \mathrm{kl}^{\prime}$ aštor

$u$ common to both German and Byelorussian: kuchmistr, mur, lancuh. Rounded vowels (vowels with umlaut) are not found in the Slavonic languages, thus German loan-words coming into Byelorussian lost this characteristic, e.g:

$\ddot{u}>u$ : štuka, uruhovati, musiti

$\ddot{o}>y:$ rychlyj

Some mhd. diphthongs have become monophthongised on their way into Byelorussian, e.g:

ei $>i$ : mistr

$a u>$ o: cofati

Consonants:

\begin{tabular}{|c|c|c|c|}
\hline$b>b$ & : biskup, balka & $h>\varnothing$ & : olstro \\
\hline$\geq p$ & : kapusta & $d>d$ & : dach \\
\hline$>v$ & : balver & $t>c$ & ricer \\
\hline$f$ & : fortka & $t>d$ & : drabina \\
\hline$f$ & : fal'šs & $t>t$ & tal'ar, trafiti $^{\prime}$ \\
\hline$>p$ & : pl'ac, pl'aga & $s>s$ & mistr, stodola \\
\hline$>g$ & : grunt, gvalt & $z, t z>c$ & : pl'ac \\
\hline $\begin{array}{l}n \\
k\end{array}$ & $\begin{array}{l}\text { : hamovati, pulhak } \\
\text { : košt krama }\end{array}$ & $\begin{array}{l}s>\dot{z}, s \\
s c h>\check{s}\end{array}$ & żegnati, ratuś \\
\hline$>c h$ & dach, kuchmistr ${ }^{12}$ & $s t>s ̌ t$ & šturm, štuka \\
\hline & puška ${ }^{13}$ & $r>l^{\prime}$ & fol'vark \\
\hline
\end{tabular}

12. H. Paul, Mittelhochdeutsche Grammatik, Tübingen, 1969, pp. 111-14.

13. P. Lessiak, Beiträge zur Geschichte des deutschen Konsonantismus, Brünn, 1933, p. 80. 
C. Morphology:

Changes in noun endings:

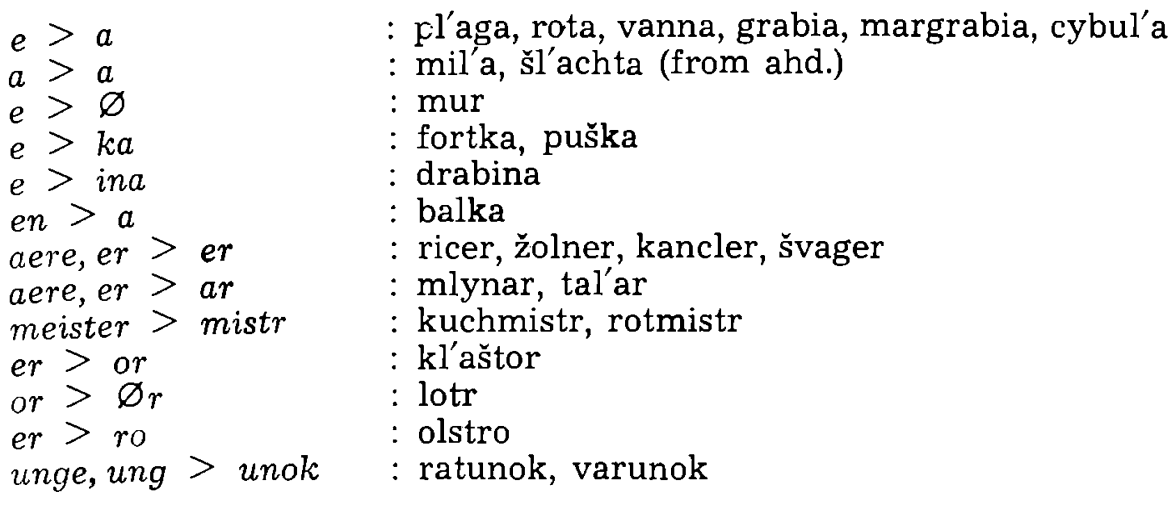

One-syllable German words taking endings in Byelorussian: rada, štuka, krama.

Compound words: ratuš, hetman, rotmistr, kuchmistr, folvark, jarmark, frimark, lancuh.

There are not many derivatives represented in the vocabulary of the two works examined in this study: biskupstvo, zbuntovanie, jarmaročnyj, gvaltovnyj, gvalčonyj, lancužno, mlynok, mlynar, morderstvo, murovanyj, prymušonyj, uradnik, ricerstvo, ricerskij, podskarbyj, neškodnyj, šl'achetnyj, šl'acht'anka, šl'achetskij, varunok, vojtovskij, zegarovyj, žolnerstvo.

Changes in verb endings:

n, en > ovati: d'akovati, folgovati, frasovati, hamovati, maršalkovati, mordovati, plundrovati, ratovati, uruhovati, šacovati, šikovati, štrafovati, švankovati

en > ati: cofati, žegnati

en $>$ iti: musiti, škoditi, trafiti

Adjectives and adverbs:

$-i v y j:$ fal'šivyj

$-n y j$ : jarmaročnyj, gvaltovnyj, gvalčonyj, koštovnyj, murovanyj, prymušonyj, neškodnyj, špetnyj

-vyj : zegarovyj

-skij : ricerskij, šl'achetskij, vojtovskij

$-l y j$ : rychlyj

In concluding this study of the German lexical borrowings in the Memoirs of Theodore Jeŭłašeŭski and the Chronicle of Barkułabava it is hoped one can see that these Germanisms did not remain 'foreign words' but have actually become part of the vocabulary of the writers of these documents, and in some cases have adapted themselves so well to their new linguistic milieu that they remain part of the Byelorussian language till today. 
Dictionartes:

\section{BIBLIOGRAPHY}

Brückner, A. Stownik etymologiczny języka polskiegu, Cracow, 1926-7.

Bułyka, A. M. Daŭntja zapazyčañni biełaruskaj movy, Minsk, 1972.

Kołas, Ja., Krapiva, K., Hlebka, P. Ruska-bielaruskı słoŭnik, Moscow, 1953.

Lexer, M. Mittelhochdeutsches Handwörterbuch, Leipzig, 1872-8.

Machek, V. Etymologický slovnik jazyka ceského a slovenského, Prague, 1957.

Matzenauer, A. Cizł slova v slovanských řečech, Brno, 1870.

Miklosich, F. Etymologisches Wörterbuch der slavischen Sprachen, Vienna, 1886.

Obrębska-Jabłońska, A., Biryła, M. Podręczny słowntk polsko-biatoruski, Warsaw, 1962.

Other works:

Bachańkoŭ, A. Ja. et. al. (eds). Histaryčnaja leksikatohija biełaruskaj movy, Minsk, 1970. Barysienka, V. V. (ed.). Historyja biełaruskaj dakastryčnickaj litaratury. Tom I. Z staražytnych casoŭ da kanca XVIII st., Minsk, 1968.

Gardiner, S. C. German Loanwords in Russian 1550-1690, Oxford, 1965.

Jurhlelevið,, P. Ja. Narys sučasnaj biełaruskaj movy z histaryčnymi kamientaryjami, Minsk, 1958.

Karnlejeva-Pletrułan, M. I. 'Mova Barkułabaŭskaha letapisu', Pracy Instytuta movaznaŭstva AN BSSR, III, Minsk, 1957.

Karskij, Je. F. Belorusy. Vvedenlje $k$ izucentju jazyka $i$ narodnoj slovesnosti, vol. I, Vilna, 1904.

Kiparsky, V. Die gemeinslavischen Lehnwörter aus dem Germanischen, Helsingfors, 1935.

Korbut, G. Wyrazy niemieckle w języku polskim, Warsaw, 1935.

Martel, A. La langue polonaise dans les pays ruthènes, Lille, 1938.

Paul, H. Mittelhochdeutsche Grammatlk, Tübingen, 1969.

Stender-Petersen, A. Slavisch-Germantsche Lehnwortkunde, Gothenburg, 1927.

Sudnik, M. P. (ed.). Bietaruskaja mova (Daśledavańni pa leksikatohii), Minsk, 1965.

Uhlenbeck, C. C. 'Germanische Wörter in Altslavischen', Archiv für slavische Philotogie, 15, Berlin.

Volski, V. Narysy pa historyi bletaruskaj litaratury epochi feadalizma, Minsk, 1958.

Zuraŭski, A. I. Historyja bletaruskaj litaraturnaj movy, I, Minsk, 1967. 